\title{
POTENTIAL SPLITTING AND NUMERICAL SOLUTION OF THE INVERSE SCATTERING PROBLEM ON THE LINE
}

\author{
Tuncay Aktosun \\ Department of Mathematics \\ North Dakota State University \\ Fargo, ND 58105 \\ Paul E. Sacks \\ Department of Mathematics \\ Iowa State University \\ Ames, IA 50011
}

\begin{abstract}
The one-dimensional Schrödinger equation is considered when the potential is real valued, integrable, has a finite first moment, and contains no bound states. From either of the two reflection coefficients of such a potential the right and left reflection coefficients are extracted corresponding to the left and right halves of the potential, respectively, and such half-line potentials are readily constructed from the extracted reflection coefficients. A computational procedure is described for such extractions and the construction of the two halves of the potential, and some applications are considered such as a numerical solution of the initial value problem for the Korteweg-de Vries equation. The theory is illustrated with some explicit examples.
\end{abstract}

PACS Numbers (1999): 02.60.Lj, 03.65.Nk

Mathematics Subject Classification (2000): 34L25, 34L40, 35Q53, 47A40, 65L09, $81 \mathrm{U} 05,81 \mathrm{U} 40$

Keywords: 1-D Schrödinger equation, Potential scattering, Inverse quantum scattering, Computational recovery of the potential, Numerical solution of the KdV equation Short title: Potential splitting in 1-D inverse scattering 


\section{INTRODUCTION}

Consider the one-dimensional Schrödinger equation

$$
\psi^{\prime \prime}(k, x)+k^{2} \psi(k, x)=V(x) \psi(k, x), \quad x \in \mathbf{R},
$$

where the prime denotes the derivative with respect to the spatial coordinate $x$, and the potential $V$ is real valued and belongs to $L_{1}^{1}(\mathbf{R})$, with $L_{1}^{1}(J)$ denoting the measurable potentials on an interval $J$ such that $\int_{J} d x(1+|x|)|V(x)|$ is finite.

The Jost solutions $f_{l}$ and $f_{r}$, from the left and right, respectively, are the solutions of (1.1) satisfying the boundary conditions

$$
\begin{gathered}
f_{l}(k, x)=e^{i k x}[1+o(1)], \quad f_{l}^{\prime}(k, x)=i k e^{i k x}[1+o(1)], \quad x \rightarrow+\infty, \\
f_{r}(k, x)=e^{-i k x}[1+o(1)], \quad f_{r}^{\prime}(k, x)=-i k e^{-i k x}[1+o(1)], \quad x \rightarrow-\infty .
\end{gathered}
$$

The transmission coefficient $T$ and the reflection coefficients from the left $L$ and from the right $R$ are related to the asymptotics of the Jost solutions as

$$
\begin{array}{ll}
f_{l}(k, x)=\frac{1}{T(k)} e^{i k x}+\frac{L(k)}{T(k)} e^{-i k x}+o(1), & x \rightarrow-\infty, \\
f_{r}(k, x)=\frac{1}{T(k)} e^{-i k x}+\frac{R(k)}{T(k)} e^{i k x}+o(1), & x \rightarrow+\infty .
\end{array}
$$

A bound state of (1.1) is a solution belonging to $L^{2}(\mathbf{R})$, and it can occur only when $k$ is on the positive imaginary axis in $\mathbf{C}^{+}$, the upper-half complex plane. We assume that no bound states exist; this is guaranteed, for example, if $V(x) \geq 0$. Under these circumstances it is well known ${ }^{1-4}$ that the potential $V$ is uniquely determined by either reflection coefficient $R$ or $L$.

Our major interest in this paper is the numerical computation of the potential from one of the reflection coefficients. In principle, this can always be done by using the FaddeevMarchenko theory via

$$
V(x)=-2 \frac{d B_{l}\left(x, 0^{+}\right)}{d x}=2 \frac{d B_{r}\left(x, 0^{+}\right)}{d x},
$$


where $B_{l}$ and $B_{r}$ are, respectively, the unique solutions of the Marchenko integral equations

$$
\begin{aligned}
& B_{l}(x, y)+\widehat{R}(2 x+y)+\int_{0}^{\infty} d z \widehat{R}(2 x+y+z) B_{l}(x, z)=0, \quad y>0, \\
& B_{r}(x, y)+\widehat{L}(-2 x+y)+\int_{0}^{\infty} d z \widehat{L}(-2 x+y+z) B_{r}(x, z)=0, \quad y>0,
\end{aligned}
$$

with

$$
\widehat{R}(y):=\frac{1}{2 \pi} \int_{-\infty}^{\infty} d k R(k) e^{i k y}, \quad \widehat{L}(y):=\frac{1}{2 \pi} \int_{-\infty}^{\infty} d k L(k) e^{i k y}
$$

This approach is not so convenient from a computational point of view because it involves solving a Fredholm-type integral equation on the semi-infinite interval $\mathbf{R}^{+}$for each value of the parameter $x$. Furthermore, the linear system obtained when either of the Marchenko equations is discretized in a straightforward way has no obvious property such as band structure to reduce the operation count. If we represent the unknown function $B_{l}$ or $B_{r}$ on a grid with $N$ points in each of the $x$ and $y$ directions, then the operation count for the recovery of $V$ in this way is $O\left(N^{4}\right)$.

On the other hand if the potential $V$ is supported in a half line, then computing solutions of the Marchenko equations becomes much easier. If, for example, $V$ vanishes for $x>x_{0}$, then it can be shown that $\widehat{R}(y)=0$ for $y>2 x_{0}$ and in the integral term in (1.2) the upper limit $+\infty$ is replaced by $2\left(x_{0}-x\right)-y$. Similarly, if $V(x)=0$ for $x<x_{0}$, then $\widehat{L}(y)=0$ for $y>-2 x_{0}$ and the upper limit $+\infty$ in the integral term of (1.3) becomes $2\left(x-x_{0}\right)-y$. An even more significant advantage in this situation is that time-domain layer-stripping methods can be used to recover the potential. These techniques do not make any direct use of the Marchenko integral equations but instead obtain $V$ directly from $\widehat{R}$ or $\widehat{L}$ in $O\left(N^{2}\right)$ operations, making use $\mathrm{u}^{5-7}$ of the hyperbolic partial differential equation satisfied by $B_{l}$ or $B_{r}$. Other fast computational techniques well suited to deal with half-line potentials have been developed in Refs. 8-10. 
We are thus motivated to ask the question whether there is any way to develop a computational method for inverse scattering on the whole line which is of comparable speed to the above mentioned methods which are available when the potential is supported in a half line. One possible approach, which is the subject of this paper, is to somehow obtain from either of the reflection coefficients of the full-line potential the right and left reflection coefficients for the left and right halves of the potential, respectively. In Section II we describe an explicit method that involves solving the Marchenko equation (1.2) or (1.3) for one $x$ value, say $x=0$, and in Section III we describe a computational algorithm based on that method. The operation count is thus $O\left(N^{3}\right)$ for this one solution of (1.2) or (1.3) and then $O\left(N^{2}\right)$ to compute the left and right halves of $V$. Thus, the net result is the reduction of the computational cost from $O\left(N^{4}\right)$ to $O\left(N^{3}\right)$. Section III also contains two numerical examples, including an application of the method to the solution

of the initial-value problem for the Korteweg-de Vries equation by the inverse scattering transform. Finally, in Section IV we briefly discuss the state of affairs when bound states are present.

\section{EXTRACTION OF HALF-LINE REFLECTION COEFFICIENTS}

Let us define

$$
V_{1}(x):=\left\{\begin{array}{ll}
V(x), & x<0, \\
0, & x>0,
\end{array} \quad V_{2}(x):= \begin{cases}0, & x<0 \\
V(x), & x>0 .\end{cases}\right.
$$

Thus $V(x)=V_{1}(x)+V_{2}(x)$, and $V_{1}$ and $V_{2}$ are supported in $\mathbf{R}^{-}$and $\mathbf{R}^{+}$, respectively. We use $R_{1}, L_{1}$, and $T_{1}$, to denote the reflection coefficient from the right, the reflection coefficient from the left, and the transmission coefficient for $V_{1}$. Similarly, by replacing the subscript 1 by 2 , we denote the corresponding quantities for $V_{2}$.

For definiteness we assume that the given scattering data is the right reflection coefficient $R(k)$. We will show that we can then uniquely determine the two half-line reflection 
coefficients $R_{1}$ and $L_{2}$, from which we may subsequently obtain $V_{1}$ and $V_{2}$, respectively. The method to recover $R_{1}$ and $L_{2}$ is quite explicit; essentially, it is necessary to solve the Marchenko equation (1.2) or (1.3) for one $x$ value, namely $x=0$ since the potential has been split at $x=0$. In a completely parallel way, or by making the change of variables $x \mapsto-x$, we may obtain $L_{1}$ and $R_{2}$ when our scattering data consists of $L$ instead of $R$.

Define the linear integral operator $\mathcal{L}$ on $L^{1}\left(\mathbf{R}^{+}\right)$as

$$
\mathcal{L} h(y):=h(y)+\int_{0}^{\infty} d z \widehat{R}(y+z) h(z), \quad y>0,
$$

and set

$$
\begin{gathered}
h(y):=B_{l}(0, y), \quad g(y):=\frac{\partial B_{l}(0, y)}{\partial x}, \\
H(k):=\int_{0}^{\infty} d y h(y) e^{i k y}, \quad G(k):=\int_{0}^{\infty} d y g(y) e^{i k y}, \\
D(k):=2 i k[1+H(k)]+G(k), \\
\xi(y):=2 \widehat{R}^{\prime}(y)+2 \int_{0}^{\infty} d z \widehat{R}^{\prime}(y+z) h(z), \quad y>0 .
\end{gathered}
$$

In the next theorem we indicate how to extract $L_{2}$ and $R_{1}$ from $R$. We use an asterisk to denote complex conjugation.

Theorem 1 Let $R$ be the reflection coefficient from the right corresponding to a real-valued potential with no bound states belonging to $L_{1}^{1}(\mathbf{R})$. Then we have

$$
L_{2}(k)=-\frac{G(k)}{D(k)}, \quad R_{1}(k)=-\frac{G(k)^{*}+R(k) D(k)}{D(k)^{*}+R(k) G(k)},
$$

in which $G$ and $D$ are defined in terms of $h$ and $g$ as above, and where $h$ and $g$ are uniquely determined from

$$
h=-\mathcal{L}^{-1} \widehat{R}, \quad g=-\mathcal{L}^{-1} \xi,
$$

with $\widehat{R}$ given in (1.4) and $\xi$ given above. 
PROOF: Let $f_{l ; 2}$ denote the Jost solution from the left for $V_{2}$ and $f_{r ; 1}$ the Jost solution from the right for $V_{1}$. Since $V_{1}=V$ for $x<0$ and $V_{2}=V$ for $x>0$, we have

$$
\begin{aligned}
& f_{r ; 1}(k, x)=f_{r}(k, x), \quad f_{r ; 1}^{\prime}(k, x)=f_{r}^{\prime}(k, x), \quad x \leq 0, \\
& f_{l ; 2}(k, x)=f_{l}(k, x), \quad f_{l ; 2}^{\prime}(k, x)=f_{l}^{\prime}(k, x), \quad x \geq 0 .
\end{aligned}
$$

Also, $V_{1}=0$ for $x>0$ implies that

$$
f_{r ; 1}(k, x)=\frac{1}{T_{1}(k)} e^{-i k x}+\frac{R_{1}(k)}{T_{1}(k)} e^{i k x}, \quad x \geq 0,
$$

and in particular

$$
f_{r ; 1}(k, 0)=\frac{1+R_{1}(k)}{T_{1}(k)}, \quad f_{r ; 1}^{\prime}(k, 0)=-i k \frac{1-R_{1}(k)}{T_{1}(k)} .
$$

Similarly

$$
f_{l ; 2}(k, 0)=\frac{1+L_{2}(k)}{T_{2}(k)}, \quad f_{l ; 2}^{\prime}(k, 0)=i k \frac{1-L_{2}(k)}{T_{2}(k)} .
$$

Combining (2.3)-(2.6) we obtain

$$
\begin{aligned}
R_{1}(k) & =\frac{i k f_{r}(k, 0)+f_{r}^{\prime}(k, 0)}{i k f_{r}(k, 0)-f_{r}^{\prime}(k, 0)}, \\
L_{2}(k) & =\frac{i k f_{l}(k, 0)-f_{l}^{\prime}(k, 0)}{i k f_{l}(k, 0)+f_{l}^{\prime}(k, 0)} .
\end{aligned}
$$

On the other hand, we have ${ }^{4,11}$

$$
f_{l}(k, x)=e^{i k x}\left[1+\int_{0}^{\infty} d y e^{i k y} B_{l}(x, y)\right]
$$

and hence

$$
f_{l}(k, 0)=1+H(k), \quad f_{l}^{\prime}(k, 0)=i k[1+H(k)]+G(k) .
$$


Using (2.8) in (2.7) we get the first equation of (2.1). Evaluating (1.2) and its derivative with respect to $x$ at $x=0$, we get $(2.2)$, where the existence and uniqueness of $\mathcal{L}^{-1}$ are assured $^{3,12}$ by the Faddeev-Marchenko theory.

To obtain the second equation of (2.1) we use the identities, see e.g. Ref. 13,

$$
\frac{1}{T(k)}=\frac{1-R_{1}(k) L_{2}(k)}{T_{1}(k) T_{2}(k)}, \quad \frac{L(k)}{T(k)}=\frac{L_{2}(k)-R_{1}(k)^{*}}{T_{1}(k)^{*} T_{2}(k)}
$$

Combining (2.9) with $R=-L^{*} T / T^{*}$, we get

$$
R(k)=\left[\frac{T_{2}(k)}{T_{2}(k)^{*}}\right] \frac{R_{1}(k)-L_{2}(k)^{*}}{1-R_{1}(k) L_{2}(k)}
$$

and solving (2.10) for $R_{1}$ we obtain

$$
R_{1}(k)=\frac{L_{2}(k)^{*} T_{2}(k)+R(k) T_{2}(k)^{*}}{T_{2}(k)+R(k) L_{2}(k) T_{2}(k)^{*}}
$$

From (2.6) and (2.8) we get

$$
T_{2}(k)=\frac{2 i k}{2 i k[1+H(k)]+G(k)}=\frac{2 i k}{D(k)} .
$$

Finally, using (2.12) and the first equation of (2.1) in (2.11), we get the second equation of $(2.1)$.

With the help of Theorem 1 , starting from $R$ we obtain successively $\widehat{R}, h, \xi, g, H, G$, $D, L_{2}$, and $R_{1}$. As mentioned earlier, $V_{1}$ and $V_{2}$ are then readily constructed from $R_{1}$ and $L_{2}$, respectively.

\section{COMPUTATIONAL PROCEDURE AND EXAMPLES}

Let us now illustrate the solution method of Theorem 1 with some numerical examples. First, we briefly elaborate the steps involved. Recall that we assume that we are given $R(k)$ and hence we suppose that $R(k)$ is known at the grid values $k_{s}:=s \Delta k$ for $s=0,1, \ldots, N$, for some chosen $N$ and $\Delta k$. 
(i) From the given data we must compute $\widehat{R}(y)$ defined in (1.4). Since $R(-k)=R(k)^{*}$ for real $k$ values, we can use the equivalent formula

$$
\widehat{R}(y)=\operatorname{Re}\left\{\frac{1}{\pi} \int_{0}^{\infty} d k R(k) e^{i k y}\right\} .
$$

Furthermore, since $\widehat{R}(y)$ is needed only for $y \geq 0$, the integral in (3.1) may be approximated by using a standard inverse discrete Fourier transform, yielding $\widehat{R}(y)$ at the grid points $y_{s}=s \Delta y$ for $s=0,1, \ldots, N$, where $\Delta y$ is determined from $\Delta k \Delta y=2 \pi / N$. In practice we may wish to replace $N$ by a larger value, say $N_{0}$, and use $\Delta k \Delta y=2 \pi / N_{0}$ so that a sufficiently small $\Delta y$ may be achieved, and for the sake of efficiency in the discrete Fourier transform we may also wish to have $N_{0}$ equal to a power of 2 . In such a case we pad with zeros for $N<s \leq N_{0}$.

(ii) Next, we solve the second-kind integral equation $\mathcal{L} h+\widehat{R}=0$ for $h$. To do this we discretize the equation as an $(M+1) \times(M+1)$ linear system

$$
h\left(y_{p}\right)+\widehat{R}\left(y_{p}\right)+\sum_{q=0}^{M} w_{q} \widehat{R}\left(y_{p}+z_{q}\right) h\left(z_{q}\right)=0, \quad p=0,1, \ldots, M,
$$

where $M$ is an integer not exceeding $N_{0}, y_{p}=z_{p}=p \Delta y$ with $\Delta y$ as in step (i), and the $w_{q}$ are some weight factors depending on the quadrature rule chosen. In the examples below we always use Simpson's rule. Recall that $\mathcal{L}$ is invertible, and this is reflected nicely in the discretization. A condition number $\kappa \approx 2$ is typical.

(iii) To obtain $\xi$ we first perform a numerical differentiation of $\widehat{R}$ by means of centered differences (and the usual one-side, three-point rule for $\widehat{R}^{\prime}(0)$ ). Now discretizing the integral operator

$$
h(y) \mapsto 2 \widehat{R}^{\prime}(y)+2 \int_{0}^{\infty} d z \widehat{R}^{\prime}(y+z) h(z),
$$

exactly as in step (ii), we get approximate values of $\xi\left(y_{p}\right)$ for $p=0,1, \ldots, M$.

(iv) Next, the approximation of $g$ may be obtained from $\xi$ in exactly the same way as $h$ from $\widehat{R}$. 
(v) Approximations of the Fourier transforms $H$ and $G$ are computed from the vectors $h\left(y_{p}\right)$ and $g\left(y_{p}\right)$ for $p=0,1, \ldots, M$ by means of the standard inverse discrete Fourier transform, padded with zeros for $M<p \leq N_{0}$ so that they are defined on a grid with the same stepsize $\Delta k$ as the original data $R(k)$.

(vi) The formulas (2.1) now immediately give us approximate values of $L_{2}\left(k_{s}\right)$ and $R_{1}\left(k_{s}\right)$ for $s=0,1, \ldots, N$.

(vii) To conclude, we use the numerical method of Ref. 7 to compute the two half-line potentials $V_{1}$ and $V_{2}$, respectively, from $R_{1}$ and $L_{2}$. If $h$ and $g$ are computed on an interval $\left[0, y_{\max }\right]$, with $y_{\max }:=M \Delta y$, features of the potential for $|x|>y_{\max } / 2$ should not be expected to be detectable - heuristically we may think of $g$ as the derivative of the Fourier transform of $L_{2}$, and it is well known that the Fourier transform of $L_{2}$ on an interval $0 \leq y \leq y_{\max }$ only determines the potential on $\left[0, y_{\max } / 2\right]$.

\section{Example 1 Let}

$$
R(k)=\frac{(k+i)(k+2 i)\left(101 k^{2}-3 i k-400\right)}{(k-i)(k-2 i)\left(50 k^{4}+280 i k^{3}-609 k^{2}-653 i k+400\right)}, \quad k \in \mathbf{R} .
$$

The corresponding potential $V$ (i.e. the unique potential without bound states for this reflection coefficient) may be computed in closed form by using one of standard techniques ${ }^{4}$ for rational scattering coefficients. We get

$$
\begin{gathered}
V_{1}(x)=\frac{16(\sqrt{2}+1)^{2} e^{-2 \sqrt{2} x}}{\left[(\sqrt{2}+1)^{2} e^{-2 \sqrt{2} x}-1\right]^{2}}, \quad x<0, \\
V_{2}(x)=\frac{96 e^{2 x}\left(81 e^{8 x}-144 e^{6 x}+54 e^{4 x}-9 e^{2 x}+1\right)}{\left(36 e^{6 x}-27 e^{4 x}+12 e^{2 x}-1\right)^{2}}, \quad x>0 .
\end{gathered}
$$

We carry out the above computational procedure with $\Delta k=.005, N=4000, N_{0}=$ $2^{17}$, and $M=800$, yielding $\Delta y \approx .01$ and $y_{\max } \approx 8$. The relative errors in $L_{2}$ and $R_{1}$ are about $11 \%$ and $6 \%$, respectively, in $L^{2}(0,20)$. These errors are largely attributable to the relatively low band limit $k_{\max }=N \Delta k=20$ and the fact that $R$ does not decay to zero 
very rapidly as $k \rightarrow \pm \infty$ in this case. If we replace $N=4000$ by $N=20000$, and hence $k_{\max }=100$, then the resulting relative errors in $L_{2}$ and $R_{1}$ are reduced to about $0.4 \%$ and $1.3 \%$, respectively. The fact that the recovery of $L_{2}$ is significantly more accurate than that of $R_{1}$, once the effects of band limitation are removed, seems to be typical, and perhaps it can be heuristically explained by the circumstance that the use of data $R$ amounts to a measurement at $x=+\infty$, so that the right-half potential $V_{2}$ may be expected to be found with smaller relative error than the left-half $V_{1}$.

Example 2 Consider the Cauchy problem for the KdV (Korteweg-de Vries) equation

$$
u_{t}-6 u u_{x}+u_{x x x}=0, \quad x \in \mathbf{R}, \quad t>0 .
$$

As is well known, ${ }^{14,15}$ if the initial state $u(x, 0)$ is given, then $u(x, t)$ may be computed by the inverse scattering transform; namely, if $R(k)$ is the reflection coefficient corresponding to the "time-independent potential" $u(x, 0)$, then the "time-evolved" scattering data $R(k) e^{8 i k^{3} t}$ corresponds to the "time-evolved potential" $u(x, t)$, which also becomes the solution of the Cauchy problem for the KdV equation at time $t$. Even if the initial state is itself supported in a half line, the same will not be true for $t>0$, so that the special computational methods for half-line potentials cited in Section I cannot be used, but the technique described above may still be applied. In other words, for each positive $t$, from the data $R(k) e^{8 i k^{3} t}$ we construct the left reflection coefficient corresponding to $\theta(x) u(x, t)$ and the right reflection coefficient corresponding to $\theta(-x) u(x, t)$, where $\theta(x)$ denotes the Heaviside function. Then, from those time-dependent, half-line reflection coefficients we construct each of the half-line potentials $\theta(x) u(x, t)$ and $\theta(-x) u(x, t)$, and thus $u(x, t)$ is obtained for all $x \in \mathbf{R}$.

In Figure 1 we show an initial state

$$
u(x, 0)= \begin{cases}\exp \left(\frac{8}{(2 x-5)(2 x-1)}\right), & |x-1.5|<1 \\ 0, & |x-1.5| \geq 1\end{cases}
$$


and the corresponding solution profile of the $\mathrm{KdV}$ equation at several later times. It is possible to create an animation of such a solution, and in fact one animation may be found at the web site http://www.math.ndsu.nodak.edu/faculty/aktosun/preprints.html.
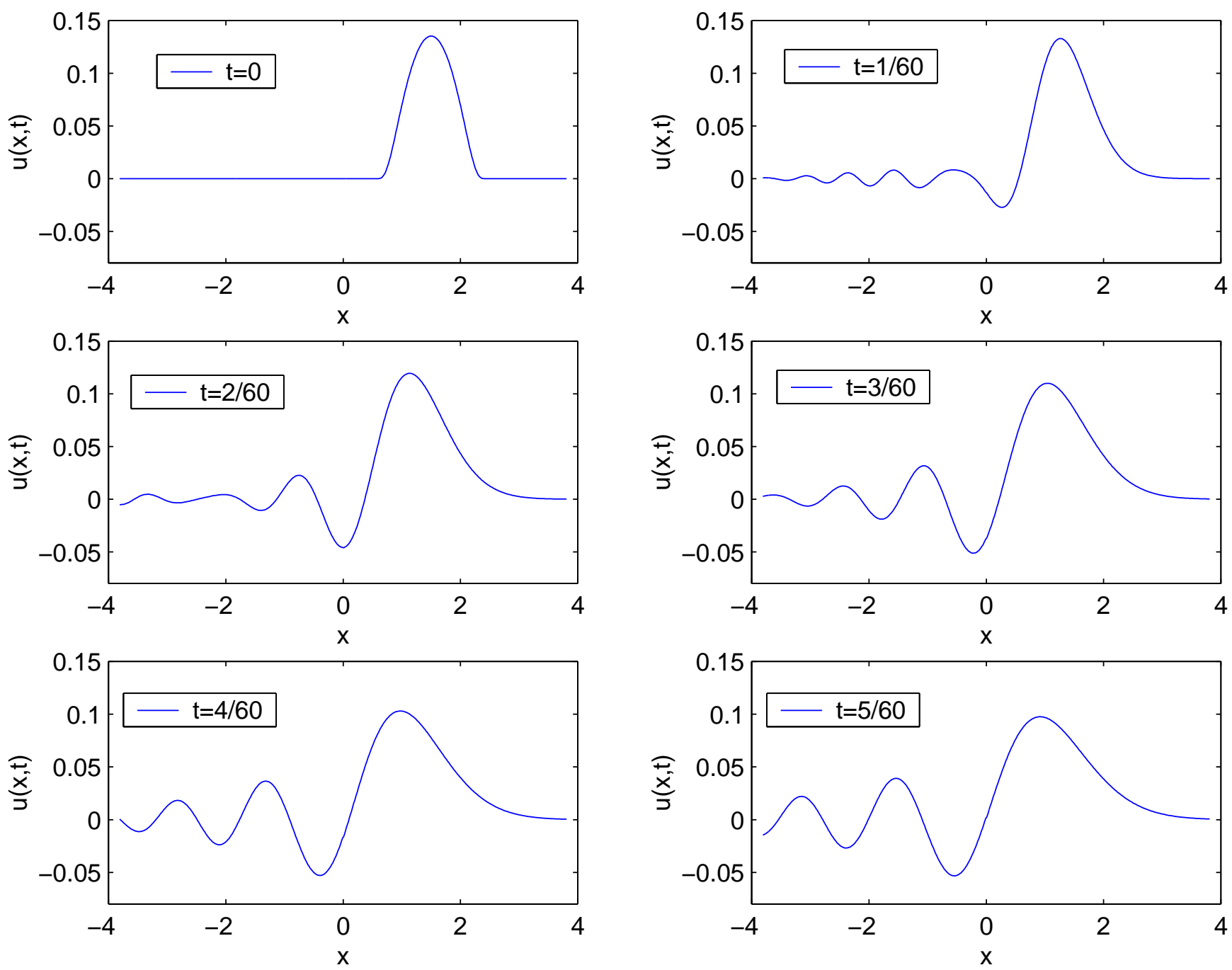

Figure 1 Some snapshots of the solution $u(x, t)$ of the KdV equation of Example 2

We should note that it is not our primary purpose here to develop an efficient numerical method to solve the initial-value problem for the $\mathrm{KdV}$ equation, and a detailed analysis of the solution method described in Example 2 is beyond the scope of this paper. There are various other numerical techniques for this purpose, e.g. see Refs. 15-18, most of which are of finite-difference or finite-element type; such methods are especially used to 
study solitary-wave (soliton) solutions of the $\mathrm{KdV}$ equation. It is well known that soliton solutions of the $\mathrm{KdV}$ equation are obtained when the initial value $u(x, 0)$ is a reflectionless potential of (1.1) with one or more bound states. In contrast, the method described in Example 2 deals with potentials having no bound states and hence non-soliton solutions of the KdV equation.

\section{CONCLUSION}

Recall that a bound state of $V$ is a solution of (1.1) which belongs to $L^{2}(\mathbf{R})$ and such states may only occur at a finite number of values $k=i \kappa_{j}$ with $j=1,2, \ldots, \mathcal{N}$, where the $\kappa_{j}$ are positive and distinct. To uniquely recover the potential $V$ in the presence of bound states, the reflection coefficient $R$ in the scattering data must be supplemented by its corresponding bound-state data, which consists of the set of $2 \mathcal{N}$ constants $\left\{\kappa_{j}, c_{j}\right\}_{j=1}^{\mathcal{N}}$ in which the $c_{j}$ are positive constants known as bound-state norming constants.

Given this scattering data, replacing $\hat{R}(y)$ by $\hat{R}(y)+\sum_{j=1}^{\mathcal{N}} c_{j}^{2} e^{-\kappa_{j} y}$ in Theorem 1 , that theorem remains valid as stated, i.e. $R_{1}$ and $L_{2}$ are determined in an unambiguous way from the data $\left\{R,\left\{\kappa_{j}, c_{j}\right\}\right\}$. In that case, the bound-state energies and norming constants for $V_{1}$ are uniquely determined by the poles and residues of the meromorphic extension of $R_{1}$ from $k \in \mathbf{R}$ to $k \in \mathbf{C}^{+}$. The situation is analogous for the bound-state data of $V_{2}$. Thus, both $V_{1}$ and $V_{2}$ are uniquely determined by $R_{1}$ and $L_{2}$, respectively, whether or not they have bound states, see e.g. Refs. 19 and 20. On the other hand, this does not help with the computational recovery of $V_{1}$ and $V_{2}$ when there are bound states. In order to numerically recover half-line potentials with bound states, to our best knowledge, no computational techniques have been developed that use only a reflection coefficient without the bound-state data.

Acknowledgments. The research leading to this article was supported in part by the National Science Foundation under grant DMS-9803219. 


\section{REFERENCES}

${ }^{1}$ L. D. Faddeev, "Properties of the $S$-matrix of the one-dimensional Schrödinger equation," Amer. Math. Soc. Transl. (Ser. 2) 65, 139-166 (1967) [Trudy Mat. Inst. Steklova 73, 314-336 (1964) (Russian)].

2 P. Deift and E. Trubowitz, "Inverse scattering on the line," Commun. Pure Appl. Math. 32, 121-251 (1979).

3 V. A. Marchenko, Sturm-Liouville Operators and Applications (Birkhäuser, Basel, 1986).

${ }^{4}$ K. Chadan and P. C. Sabatier, Inverse Problems in Quantum Scattering Theory, 2nd ed. (Springer, New York, 1989).

5 W. Symes, "Inverse boundary value problems and a theorem of Gel'fand and Levitan," J. Math. Anal. Appl. 71, 379-402 (1979).

${ }^{6}$ A. Bayliss, Y. Li, and C. S. Morawetz, "Scattering by a potential using hyperbolic methods," Math. Comp. 52, 321-338 (1989).

7 P. E. Sacks, "Reconstruction of steplike potentials," Wave Motion 18, 21-30 (1993).

8 Y. Chen and V. Rokhlin, "On the inverse scattering problem for the Helmholtz equation in one dimension," Inverse Problems 8, 365-391 (1992).

9 J. H. Rose, "Global minimum principle for one-dimensional inverse scattering with bound states," Inverse Problems 13, L1-L5 (1997).

10 J. Sylvester and D. P. Winebrenner, "Linear and nonlinear inverse scattering," SIAM J. Appl. Math. 59, 669-699 (1999).

11 R. G. Newton, "Inverse scattering. I. One dimension," J. Math. Phys. 21, 493-505 (1980). 
12 T. Aktosun, M. Klaus, and C. van der Mee, "Scattering and inverse scattering in one dimensional nonhomogeneous media," J. Math. Phys. 33, 1717-1744 (1992).

13 T. Aktosun, "A factorization of the scattering matrix for the Schrödinger equation and for the wave equation in one dimension," J. Math. Phys. 33, 3865-3869 (1992).

14 P. Lax, "Outline of a theory of the KdV equation," In: T. Ruggeri (ed.), Recent mathematical methods in nonlinear wave propagation, 70-102, Lecture Notes in Math. 1640 (Springer, Berlin, 1996).

15 M. J. Ablowitz and H. Segur, Solitons and the inverse scattering transform (SIAM, Philadelphia, 1981).

16 J. L. Bona, V. A. Dougalis, O. A. Karakashian, and W. R. McKinney, "Conservative, high-order numerical schemes for the generalized Korteweg-de Vries equation," Philos. Trans. Roy. Soc. London Ser. A 351, 107-164 (1995).

17 T. R. Taha and M. J. Ablowitz, "Analytical and numerical aspects of certain nonlinear evolution equations: III. Numerical, Korteweg-de Vries equation," J. Comput. Phys. 55, 231-253 (1984).

18 A. Provenzale and A. R. Osborne, "Nonlinear Fourier analysis for the infinite-interval Korteweg-de Vries equation. II. Numerical tests of the direct scattering transform," J. Comput. Phys. 94, 314-351 (1991).

19 N. N. Novikova and V. M. Markushevich, "Uniqueness of the solution of the onedimensional problem of scattering for potentials located on the positive semiaxis," Comput. Seismology 18, 164-172 (1987) [Vychislitel'naya Seismologiya 18, 176-184 (1985) (Russian)].

20 T. Aktosun, "Bound states and inverse scattering for the Schrödinger equation in one dimension," J. Math. Phys. 35, 6231-6236 (1994). 